\title{
Analysis of the Specific Heat for the Transitions of the Phases I-II-III-IV in $\left(\mathrm{N}\left(\mathrm{CH}_{3}\right)_{4}\right)_{2} \mathrm{CuCl}_{4}$ Crystal
}

\author{
H. Yurtseven ${ }^{*}$ and I. Yardımcı \\ Department of Physics, \\ Middle East Technical University \\ 0653I Ankara- TURKEY
}

(Received August 5, 2008: final form August 12, 2008)

\begin{abstract}
The temperature dependence of the specific heat $C_{p}$ is analyzed here using the experimental data according to a power-law formula with the critical exponent $\alpha$ for the transitions of commensurate (I), incommensurate (II) and the ferroelastic (III and IV) phases in $\left(\mathrm{N}\left(\mathrm{CH}_{3}\right)_{4}\right)_{2} \mathrm{CuCl}_{4}$ crystal. From our analysis, we extract the value of $\alpha \approx 0$ above and below $T_{c}$ for the successive phase transitions of I-II-III, which is expected from the mean field theory ( $\alpha=0$ discontinuity), XY model ( $\alpha=0$ $\log )$ and the two dimensional Ising model $(\alpha=0 \log )$. This indicates that the transitions between commensurate and incommensurate phases and also between incommensurate and ferroelastic (III) phases in TMATC-Cu are of a second order type.

It is indicated here that the transition between the ferroelastic phases III and IV in TMATC-Cu should be further investigated using theoretical models.
\end{abstract}

Key Words: Incommensurate, ferroelastic, TMATC$\mathrm{Cu}$.

\section{INTRODUCTION}

$\left(\mathrm{N}\left(\mathrm{CH}_{3}\right)_{4}\right)_{2} \mathrm{CuCl}_{4}$ crystal is the copper compound (TMATC-Cu) of the $\mathrm{A}_{2} \mathrm{BX}_{4}$ compounds where $A$ denotes tetra-alkylammonium group, $B$ and $X$ are the transition metal and halogen, respectively. These compounds exhibit successive phase transitions with an incommensurate phase close to the room temperature /1-4/. They also exhibit a ferroelastic phase at low temperatures $/ 3-5 /$.

Elastic properties of ferroelectric with incommensurately modulated structure have been studied by ultrasonic method /6/. For modulated phases of dielectric $\mathrm{A}_{2} \mathrm{BX}_{4}$ family, a symmetry-based phenomenological model has been developed $/ 7,8$. As the layered structures, the free energy has been expanded in terms of the amplitudes of the two symmetry modes, and the space groups and the modulation wave vectors have been predicted for the successive phase transitions in the $\mathrm{A}_{2} \mathrm{BX}_{4}$ compounds 17, 8\%. Also, a crystallographic study of the five compounds of the $\mathrm{Cs}_{2} \mathrm{BX}_{4}$ family with $\mathrm{B}=\mathrm{Zn}, \mathrm{Cu}$ and $\mathrm{X}=\mathrm{Cl}, \mathrm{Br}$ has been reported 19/. Experimentally, regarding the $\left(\left(\mathrm{CH}_{3}\right)_{4} \mathrm{~N}\right)_{2} \mathrm{MX}_{4}$ salts, phase transitions and lattice parameters of sixteen $\left(\left(\mathrm{CH}_{3}\right)_{4} \mathrm{Z}\right)_{2} \mathrm{MX}_{4}(\mathrm{Z}=\mathrm{P}$, As, $\mathrm{Sb} ; \mathrm{M}=\mathrm{Co}, \mathrm{Cu}, \mathrm{Zu} ; \mathrm{X}=\mathrm{Cl}, \mathrm{Br}, \mathrm{I})$ compounds have been studied $/ 10 \%$, which all undergo a high-temperature, $\beta-\mathrm{K}_{2} \mathrm{SO}_{4}$ structure type $/ 11,12 \%$. As an extension of this $\left(\left(\mathrm{CH}_{3}\right)_{4} \mathrm{~N}\right)_{2} \mathrm{MX}_{4}$ family, or (TMA) $)_{2} \mathrm{MX}_{4}$ family where TMA is the tetramethylammonium, their deuterated analogs have also been studied $/ 12 \%$.

It has been indicated that in the incommensurate

\footnotetext{
- Corresponding author

e-mail: hamit@metu.edu.tr
} 
phase (II) the modulation wave vector is approximately constant $/ 13 /$. Hence, the three-dimensional lattice translational symmetry is broken in this phase, whereas in the lock-in phase (III) the modulation becomes commensurate with the lattice, as also indicated previously $/ 16 /$. Thus, this lock-in phase is ferroelectric since it has three-dimensional lattice translational symmetry, which becomes ferroelastic for tetramethylammonium tetrachlorocuprate $/ 14 /$. As the temperature decreases, the lock-in phase (III) transforms into another lock-in phase (IV) which is also a ferroelastic $/ 17 /$. The three successive phases (I-II-IIIIV) have been detected by thermal, optical and spectroscopic measurements for TMATC-Cu /18/. In particular, the influence of incommensurate modulation on the optical properties of this compound has been studied experimentally $/ 16 /$.

TMATC-Cu in particular exhibits three successive transitions /13-15/ from commensurate (I) to incommensurate (II) at $298 \mathrm{~K}$, from incommensurate (II) to ferroelastic (III) at $293 \mathrm{~K}$ and from ferroelastic (III) to ferroelastic (IV) at $265 \mathrm{~K}$. The commensurate (I) or normal phase has the orthorhombic structure with the space group $D_{2 h}^{16}$, whereas the ferroelastic phases III and IV have the monoclinic structures with the space group $C_{2 h}^{5}$. Both monoclinic phases III and IV are different in their orientational domains.

As reported in the experimental studies on TMATC$\mathrm{Zn}$ and TMATC-Co crystals $/ 19,20 /$ which exhibit transitions associated with organic group movements at low temperatures, TMATC-Cu also exhibits an orderdisorder transition. Above the room temperature, it has been indicated on the basis of the measurements of the specific heat $C_{p} / 18 /$ that those three compounds have almost the same disordered phase. Also, it has been reported /18/ that the Raman measurements give the transition from commensurate (I) to ferroelastic (IV) as a first order, in particular the critical behavior of the commensurate (I) to incommensurate (II) as a second order type. It has been indicated $/ 18 /$ that lock-in transition between the incommensurate (II) and ferroelastic (III) phases is induced due to a combination of improper ferroelasticity (III) and incommensurate (II)-commensurability (I).

It has also been indicated that from the incommensurate (II) phase to the lock-in phase, a large thermal hysteresis (nearly $7 \mathrm{~K}$ ) has been detected in the measurement of the linear birefringence $116 /$, as observed (about 10K) in the measurements of the dielectric constant $/ 14 /$. It has been obtained that near the incommensurate (II) to commensurate improper ferroelastic phase (III), the elastic constant exhibits similar anomalous behavior as the dielectric constant for $\left(\left(\mathrm{CH}_{3}\right)_{4} \mathrm{~N}\right)_{2} \mathrm{CuCl}_{4}$, which has been explained $/ 21 /$ using the soliton theory $122 \%$. On the other hand, the elastic anomalies obtained at ultrasonic frequencies $(10 \mathrm{MHz})$ near the paraelectric (I)-incommensurate (II) transition in this compound have been explained using the planewave approximation $121 \%$. By relating the thermodynamic potentials among the phases, a P-T phase diagram for a $\left(\left(\mathrm{CH}_{3}\right)_{4} \mathrm{~N}\right)_{2} \mathrm{CuCl}_{4}$ crystal has been calculated $/ 23 /$.

Regarding the three successive phase transitions (III-III-IV) that occur in TMATC-Cu, the measurements of the specific heat $C_{p}$ have been carried out by adiabatic calorimetry and the specific heat data has been analyzed for the critical behavior of commensurate (I) incommensurate (II) phase transition $/ 18 /$, as we stated above.

In this study we investigate the order-disorder mechanism for the commensurate (I)-incommensurate (II)-ferroelastic (III to IV) phase transitions in TMATC$\mathrm{Cu}$ by analyzing the experimental data for the specific heat $C_{p} / 18 /$ according to a power-law formula. From our analysis, we deduce the values of the critical exponent $\alpha$ for the specific heat $C_{p}$ above and below the transition temperature $T_{c}$ for all the successive transitions I-II-III-IV in TMATC-Cu. Our $a$ values are compared with those obtained previously and also with the predictions of the mean field theory, $\mathrm{XY}$ and the Ising models.

\section{CALCULATIONS AND RESULTS}

We analyzed in this study the experimental data which was obtained by the calorimetric measurements for the $\left(\mathrm{N}\left(\mathrm{CH}_{3}\right)_{4}\right)_{2} \mathrm{CuCl}_{4} / 18 /$ close to the phase transitions (I-II-III-IV). For this analysis, we used the power law formula 


$$
C_{p} / \mathrm{R}=A\left(T-T_{c}\right)^{-\alpha}
$$

with the critical exponent $\alpha$ and the amplitude $A$ for the specific heat $C_{p}$. Here $\mathrm{R}$ is the gas constant (8.314 $\mathrm{J} / \mathrm{mol}$.K). Our analysis was performed below and above the transition temperature $T_{c}$ for all the phases, I-II-III and IV in TMATC-Cu. By taking the logarithm of both sides in Eq. (1), we have

$$
\log \left(C_{p} / \mathrm{R}\right)=\log A-\alpha \log \left(T-T_{c}\right)
$$

So, a linear plot of $C_{p} / \mathrm{R}$ against $\left|T-T_{c}\right|$ in the $\log -\log$ scale gives the slope value of $\alpha$ and the intercept $\log A$.

From our analysis of the transitions between the phases I-II, II-III and III-IV using the experimental data for the specific heat $C_{p} / \mathrm{R}$ in TMATC-Cu /18/, we deduced the values of the critical exponent $\alpha$ and the amplitude $A$. For the analysis of all the transitions studied, the values of $\alpha$ and $A$ with the regions of the reduced temperature $\varepsilon=\left|\left(T: T_{c}\right) / T_{c}\right|$ are presented in Tables 1 and 2 above and below $T_{c}$, respectively. Figs. (1-3) give the specific heat $C_{p} / \mathrm{R}$ in a $\log -\log$ scale as a function of temperature for the transitions of I-II, II-III and III-IV of TMATC-Cu, respectively in the temperature intervals indicated, as also tabulated in Tables 1 and 2. We give here one plot for each transition to represent the specific heat $C_{p}$ as a function of the temperature for TMATC-Cu. The other plots of $C_{p} / \mathrm{R}$ against the temperature which we obtained for the transitions I-II, II-III and III-IV, in the temperature intervals indicated in Tables 1 and 2, are similar to Figs. (1-3) given here.

\section{DISCUSSION}

The analysis of the specific heat $C_{p}$ was performed here to describe the critical behavior of the transitions among the commensurate (I), incommensurate (II) and ferroelastic (III and IV) phases in TMATC-Cu crystal according to a power-law formula (Eq. 1). From our analysis, we extracted the value of the critical exponent as $\alpha \sim 0$ above and below $T_{c}$ for the commensurate (I)incommensurate (II) transition $\left(T_{c}=298 \mathrm{~K}\right)$ except in the temperature range of $T \cdot T_{c}=0$ and $0.4 \mathrm{~K}$ where we obtained the value of $\alpha=-0.32$, as given in Tables 1 and
2. This negatively large value of the critical exponent $\alpha$ may be due to fluctuations in the vicinity of the $T_{c}$, as one approaches the critical point from above. Our value of $\alpha \approx 0$ was also obtained above and below $T_{c}$ by the analysis of the excess specific heat $\Delta C_{p}$ for the I-II transition in a relatively small temperature range of $0.005 \mathrm{~K}<T_{l}-T<0.04 \mathrm{~K} / 18 /$. The near zero value of the critical exponent for the specific heat which was obtained here is the same as the mean field value ( $\alpha=\alpha^{\prime}$ $=0)$ and also that predicted from the XY model ( $\alpha=0$, $\log )$ and the two dimensional Ising model $(\alpha=0, \log )$. This indicates that the commensurate (I)incommensurate (II) transition is of a second order.

Considering the transition from incommensurate (II) phase to the ferroelastic (III) phase, which occurs at 293 $K$, our value of the critical exponent was $\alpha \approx 0$ within the same temperature interval above and below $T_{c}$ (Tables 1 and 2). This also indicates that the transition between the phases II and III is of a second order as the transition between the phases I and II. This is supported by the small enthalphic value occurring at the transition between the incommensurate (II) and the ferroelastic (III) phases in comparison with the enthalphic values for the I-II and III-IV transitions in TMATC-Cu /18/. Furthermore, from the birefringence measurements for the transition between the phases II and III, the temperature dependence of the order parameter was analyzed according to a power-law formula and the value of $\beta=0.16 \pm 0.03$ was extracted as the critical exponent for the order parameter of phase II in TMATC-Cu /18/. This value of $\beta$ is close within the uncertainties to the value of $1 / 8$ predicted by an Ising model $(d=2, n=1)$, for a second order phase transition.

As the negative values except the value of $\alpha \sim 0.1$ in the temperature range of 0.2 to $0.3 \mathrm{~K}$, we extracted the values of the critical exponent $\alpha$ for the specific heat $C_{p}$, which vary from 0.2 to 1.0 above and below $T_{c}$ for the transition between the ferroelastic phases III and IV, as given in Tables 1 and 2. Those values are considerably large in comparison with the mean field value or the model predictions $\left(\alpha=\alpha^{\prime}=0\right)$. Our $\alpha$ values deduced for the III-IV transition in TMATC-Cu can possibly be better compared with the prediction of the Heisenberg $(\mathrm{d}=3, \mathrm{n}=3)$ model as designated for TMATC-Zn /10/ and $\mathrm{K}_{2} \mathrm{SeO}_{4} / 11 \%$. 
Table 1

Values of the critical exponent $\alpha$ and the amplitude $A$ using the experimental data for the specific heat $C_{p} / 9 /$ of TMATC-Cu according to the power-law relation (Eq.

1) for the transitions indicated in the temperature interval above $T_{c}$.

\begin{tabular}{|c|c|c|c|c|}
\hline Transition & $\begin{array}{c}\mathrm{T}_{\mathrm{c}} \\
(\mathrm{K})\end{array}$ & $-\alpha$ & $A$ & $\varepsilon=\left(T_{-}-T_{c}\right) / T_{\mathrm{c}}$ \\
\hline \multirow{3}{*}{ I-II } & \multirow{2}{*}{298} & 0.03 & 57.55 & $\begin{array}{c}1.3 \times 10^{-3}<\varepsilon< \\
8.4 \times 10^{-3}\end{array}$ \\
\cline { 3 - 5 } & & 0.32 & 59.38 & $0<\varepsilon<1.3 \times 10^{-3}$ \\
\hline II-III & 293 & 0.004 & 59.55 & $0<\varepsilon<4.1 \times 10^{-3}$ \\
\hline \multirow{3}{*}{ III-IV } & \multirow{2}{*}{265} & 0.19 & 55.27 & $\begin{array}{c}2.5 \times 10^{-3}<\varepsilon< \\
6.0 \times 10^{-3}\end{array}$ \\
\cline { 3 - 5 } & & 1.02 & 27.23 & $\begin{array}{c}1.9 \times 10^{-3}<\varepsilon< \\
2.5 \times 10^{-3}\end{array}$ \\
\cline { 3 - 5 } & & 0.21 & 90.31 & $\begin{array}{c}7.5 \times 10^{-4}<\varepsilon< \\
1.9 \times 10^{-3}\end{array}$ \\
\hline
\end{tabular}

\section{Table 2}

Values of the critical exponent $\alpha$ and the amplitude $A$ using the experimental data for the specific heat $C_{p}$ /9/ of TMATC-Cu according to the power-law relation (Eq.

1) for the transitions indicated in the temperature interval below $T_{c \text {. }}$.

\begin{tabular}{|l|l|c|l|l|}
\hline Transition & $\begin{array}{l}\mathrm{T}_{\mathrm{c}} \\
(\mathrm{K})\end{array}$ & $\alpha$ & $A$ & $\varepsilon=\left(T_{c}-T\right) / T_{\mathrm{c}}$ \\
\hline \multirow{2}{*}{ I-II } & 298 & 0.01 & 63.5 & $0<\varepsilon<5.0 \times 10^{-3}$ \\
\cline { 3 - 5 } & -0.02 & 61.3 & $\begin{array}{l}5.0 \times 10^{-3}<\varepsilon< \\
1.6 \times 10^{-2}\end{array}$ \\
\hline II-III & 293 & -0.01 & 58.5 & $0<\varepsilon<4.1 \times 10^{-3}$ \\
\hline III-IV & 265 & -0.59 & 61.1 & $0<\varepsilon<7.5 \times 10^{-4}$ \\
\cline { 3 - 5 } & & 0.09 & 45.6 & $\begin{array}{l}7.5 \times 10^{-4}<\varepsilon< \\
1.1 \times 10^{-3}\end{array}$ \\
\cline { 3 - 5 } & & -0.19 & 51.7 & $\begin{array}{l}1.1 \times 10^{-3}<\varepsilon< \\
3.0 \times 10^{-3}\end{array}$ \\
\hline
\end{tabular}

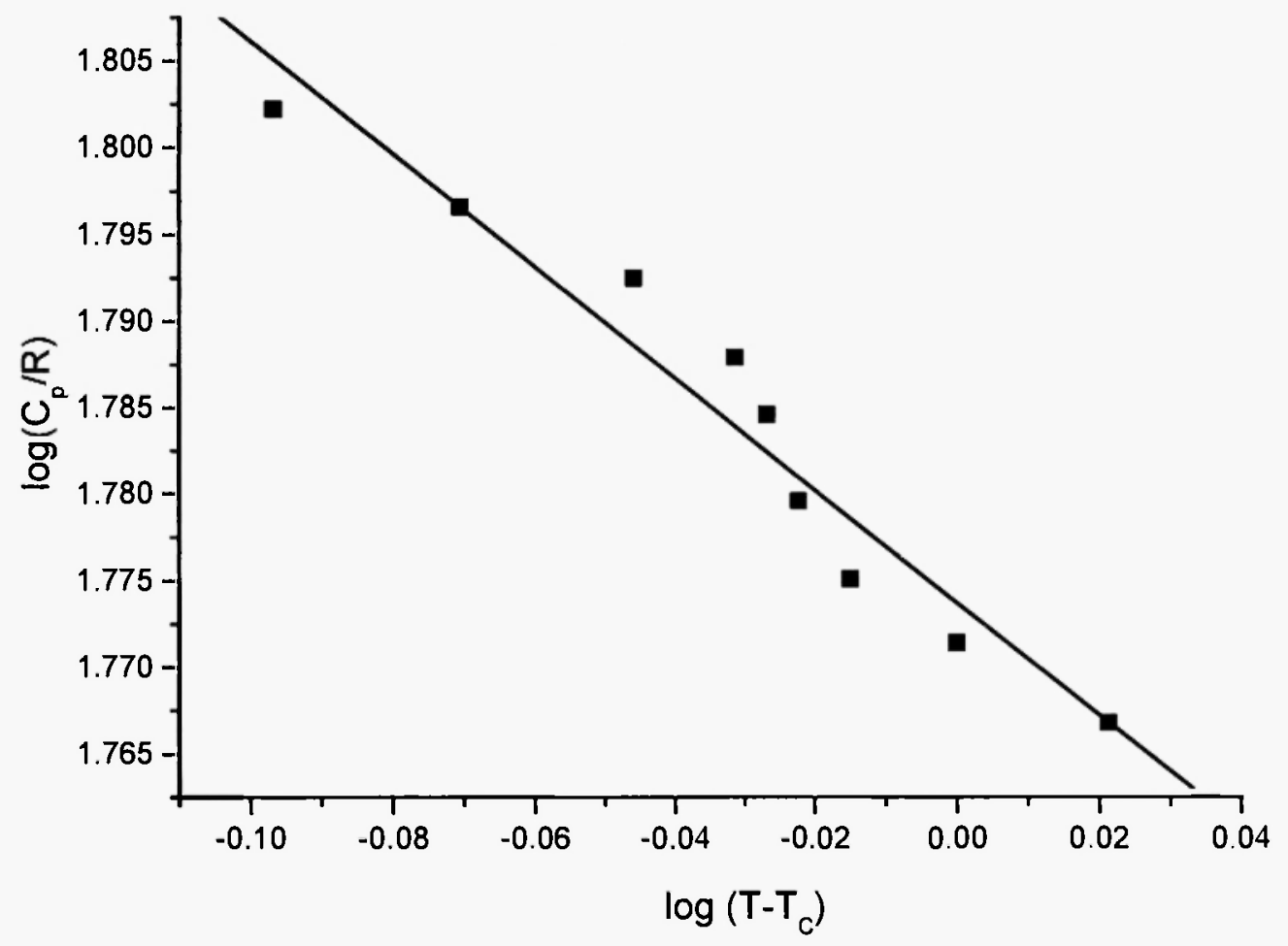

Fig. 1: Specific heat of TMATC-Cu in a log-log scale as a function of temperature for the I-11 phase transition in the temperature interval of $298.8 \mathrm{~K}<T .<299.2 \mathrm{~K}\left(T_{c}=298 \mathrm{~K}\right)$ according to a power-law relation $(\mathrm{Eq} . \mathrm{l})$. $\mathrm{R}$ denotes the gas constant. 


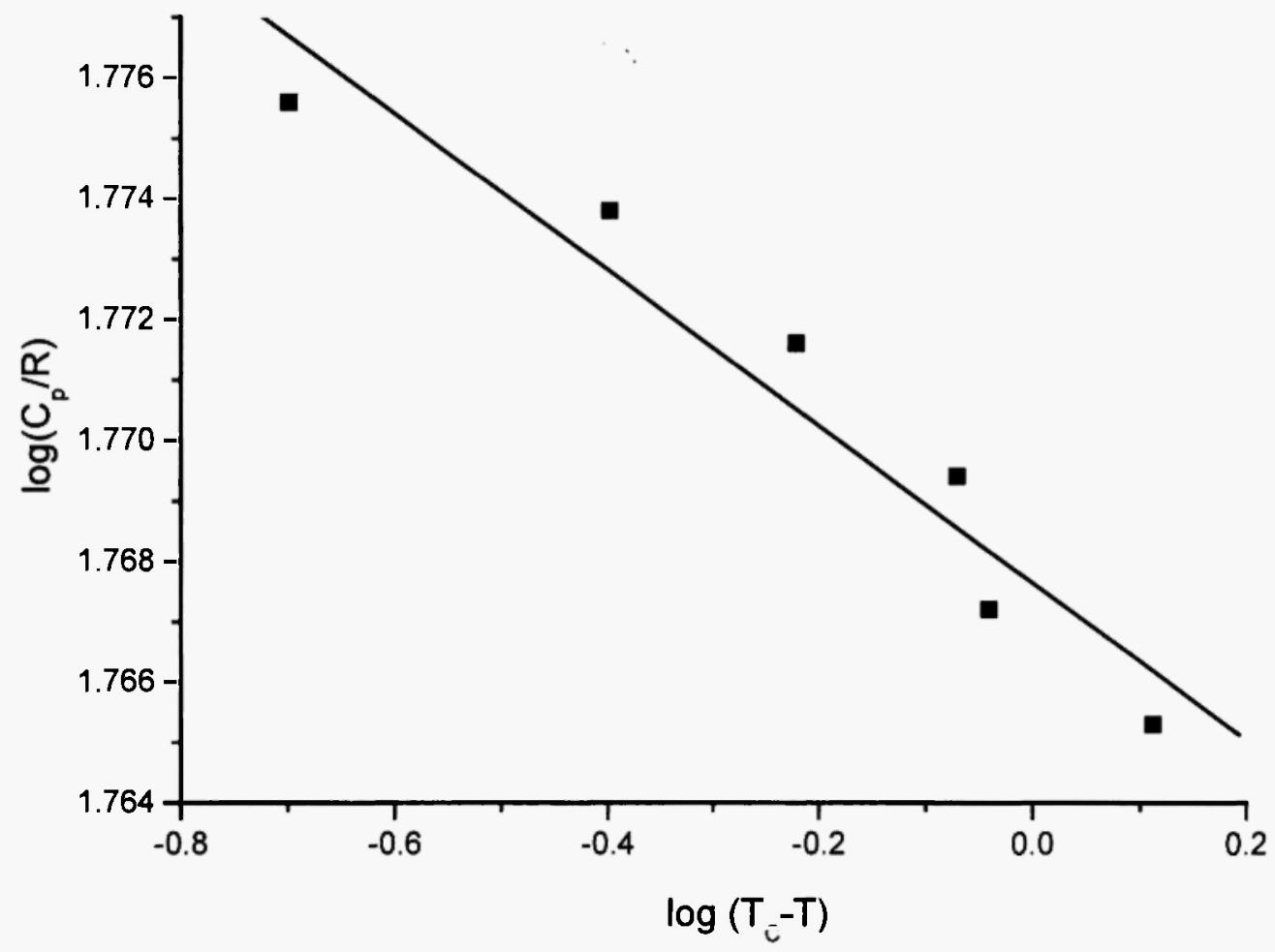

Fig. 2: Specific heat of TMATC-Cu in a $\log -\log$ scale as a function of temperature for the 11-III phase transition in the temperature interval of $291.4 \mathrm{~K}<T<299.7 \mathrm{~K}\left(T_{c}=293 \mathrm{~K}\right.$ ) according to a power-law relation (Eq. 1). R denotes the gas constant.

\section{CONCLUSIONS}

We studied here the temperature dependence of the specific heat $C_{p}$ for the transitions among the phases of the commensurate (I), incommensurate (II) and the ferroelastic (III and IV) in $\left(\mathrm{N}\left(\mathrm{CH}_{3}\right)_{4}\right)_{2} \mathrm{CuCl}_{4}$ crystal. By analyzing the experimental $C_{p}$ data according to a power-law formula, the values of the critical exponent $a$ were extracted above and below $T_{c}$ for the transitions studied here. On the basis of our exponent value of $\alpha=$ 0 , which is expected from the mean field theory, the transitions among the phases I, II and III are of a second order.

It is suggested here that the transition between the phases III and IV can be treated adequately with the Heisenberg model $(\alpha=-0.08 \pm 0.04)$.

\section{REFERENCES}

1. M. Iizumi, J. D. Axe, G. Shirane and K. Shimakoa, Phys. Rev. B 15, 4392-4411 (1977).

2. K. Gesi and M. Iizumi, J. Phys. Soc. Jpn. 45, 1777-1778 (1978).

3. S. Sawada, Y. Shiroishi and A. Yamamoto, Ferroelectrics 21, 413-414 (1978).

4. S. Sawada, Y. Shiroishi, A. Yamamoto, M. Takashige and M. Matsuo, Phys. Lett. A 67, 56-58 (1978).

5. S. Sawada, Y. Shiroishi, A. Yamamoto, M. Takashige and M. Matsuo, J. Phys. Soc. Jpn. 44, 687-688 (1978).

6. V.V. Lemanov, Ferroelectrics 78, 163-I72 (1988).

7. Z. Y. Chen and M. B. Walker, Phys. Rev. Lett. 65, 


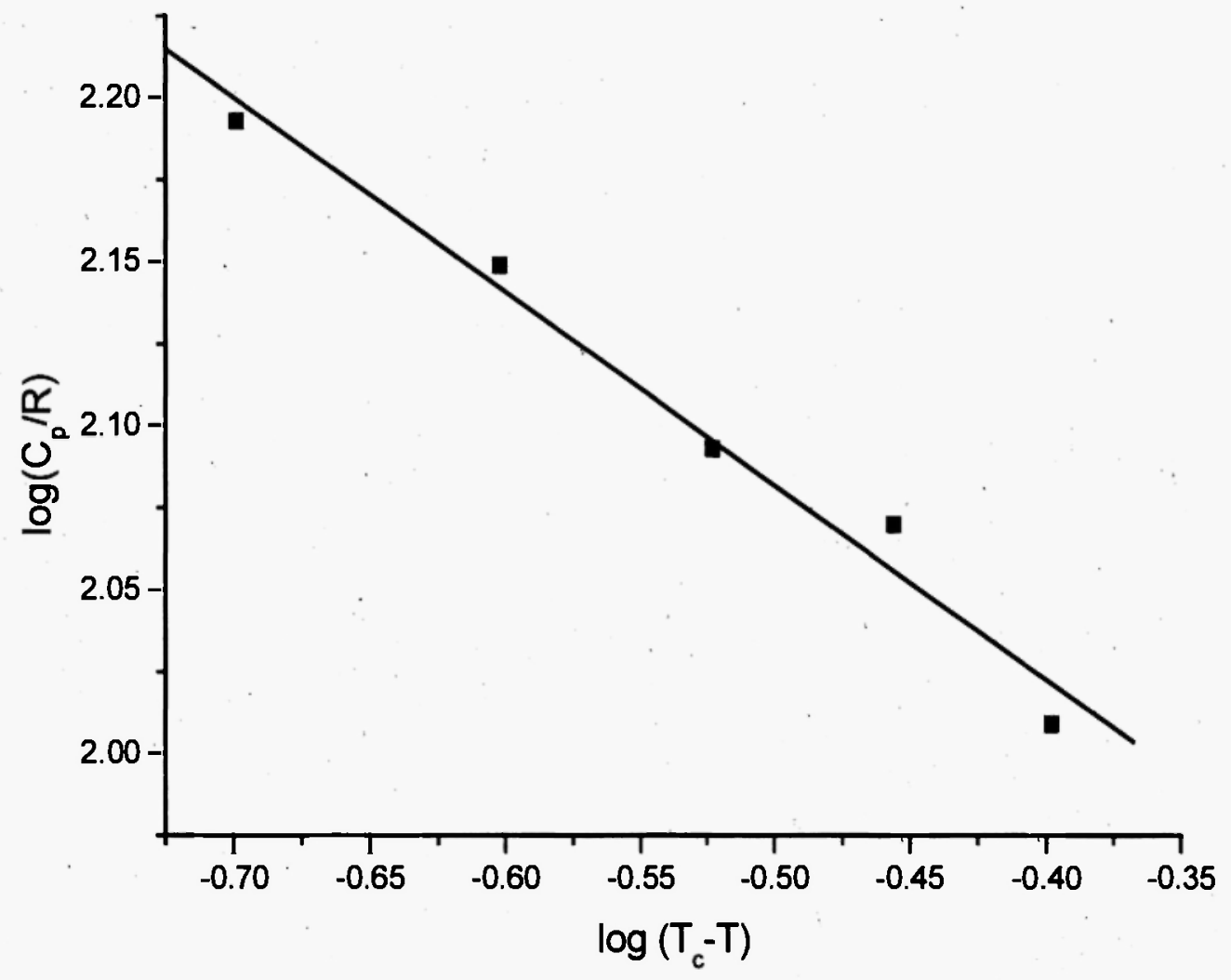

Fig. 3: Specific heat of TMATC-Cu in a $\log -\log$ scale as a function of temperature for the III-VI phase transition in the temperature interval of $264.6 \mathrm{~K}<T<265 \mathrm{~K}\left(T_{c}=265 \mathrm{~K}\right)$ according to a power-law relation (Eq. 1). $\mathrm{R}$ denotes the gas constant.

1223-1226 (1990).

8. Z. Y. Chen and M. B. Walker, Phys. Rev. B 43 5634-5648 (1991).

9. R. Puget, M. Tannin, R. Perret, L. Godefroy and G. Godefroy, Ferroelectrics 107, 229-234 (1990).

10. M. R. Pressprich, M. R. Bond and R.D. Willett, Phys. Rev. B 43, 13549-13558 (1991).

11. O. Müller and R. Roy, The Major Ternary Structural Families, Springer, New York, 1974.

12. R. Peret, G. Godefroy and H. Arend, Ferroelectrics 73, 87-99 (1987).

13. K. Gesi and M. lizumi, J. Phys. Soc. Jpn. 48, 1775-1776 (1980).

14. A. Sawada, J. Sugiyama, W. Wada and Y. Ishibashi, J. Phys. Soc. Jpn. 48, 1773-1774 (1980).

15. A. Sawada, J. Sugiyama, W. Wada and Y. Ishibashi, J. Phys. Soc. Jpn. 49, 89-91 (1980).
16. M. Kremers and H. Meekes, J. Phys: Condens. Matter 7, 8119-8138 (1995).

17. J. Sugiyama, W. Wada, A. Sawada and Y. Ishibashi, J. Phys. Soc. Jpn. 49, 1405-1412 (1980).

18. A. Gomez-Cuevas, M. J. Tello, J. Fernandez, A. Lopez-Echarri, J. Herreros and M. Couzi, J. Phys. C: Solid State Phys. 16, 473-485 (1983)

19. I. Ruiz-Larrea, A. Lopez-Echarri and M. J. Tello, J. Phys. C: Solid State Phys. 14, 3171-3176 (1981).

20. A. Lopez-Echarri, M. J. Tello and P. Gilli, Solid State Commun. 36, 1021-1025 (1980).

21. A. V. Kityk, V. P. Soprunyuk, A. Fuith, W. Schranz and H. Warhanek, Phys. Rev. B 53, 6337. 6344 (1996).

22. J. Holakovsky and V. Dvorak, J. Phys. C: Solid State Phys. 21, 5449-5454 (1988).

23. D. G. Sannikov, Phys. of Solid State 42, 2282$2287(2000)$. 